\title{
Relapsing Brucellosis due to the Infection of Implanted Cardiac Device
}

The Editor,

Sir,

The involvement of implanted electronic cardiac devices by haematogenous seeding of brucellosis is quite rare. To our knowledge, nearly 10 cases of pacemaker and implantable cardioverter-defibrillator (ICD) infection and a few cases of transvenous lead endocarditis caused by brucella has been described in the literature. We present a case of relapsing brucellosis due to the infection of the generator pocket and transvenous leads by Brucella melitensis. Implanted cardiac pacemaker was a localized foci of infection that caused relapses. Brucellosis was resolved only after the removal of the pacemaker and leads and subsequent appropriate antibiotic therapy.

A 56-year-old male patient who had been implanted a pacemaker for bradycardia in 2003, presented to a state hospital in August, 2010, with an acute febrile illness and the complaints of night sweats, fever and weakness. In his blood culture, Brucella melitensis was isolated. It was acquired from his consumption of unpasteurized cow's milk. He was administered rifampicin and doxycycline for six weeks. But very soon, his fever relapsed. He was diagnosed as B melitensis relapse at another state hospital and was treated by rifampicin and doxycycline antibiotics for six weeks and streptomycin for 21 days, and his symptoms improved. Just after 20 days of the second therapy, his fever relapsed once more. The patient was then readmitted for the recurrence of brucellosis. This time, brucella serology with standard tube agglutination (STA) was positive in titer of 1:2560, and in his blood culture, and B melitensis was isolated again. Because of frequent relapses, the generator pocket was suspected to be the responsible focus for the relapses although there was no sign of inflammation and any purulent material in the pacemaker pocket region. The patient was then referred to our centre for further investigation of the generator pocket and the leads and possible surgical removal of the pacemaker. The patient was acknowledged for the potential risks of the removal of a permanent pacemaker, but the patient denied the therapy and was discharged subsequently. Then he was treated intermittently with rifampicin and doxycycline for three years for relapses. After three years, the patient presented with fever again. Soft-tissue ultrasound demonstrated fluid in the generator pocket. Brucella melitensis was isolated from the blood cultures of the samples taken from the pacemaker pocket. He was then referred to our centre for the surgical removal of the pacemaker generator and leads and additional drainage of the pacemaker pocket. Transthoracic and transesophageal echocardiography showed no cardiac vegetation. The patient was pacemaker independent; so, the pacemaker generator and leads were removed and cultured. Brucella melitensis was isolated from the cultures of the pus and necrotic tissue from the pacemaker pocket. Brucella was also cultured from the lead tips confirming the diagnosis of transvenous lead endocarditis. Antibiotic treatment consisted of a 45-day regimen of $200 \mathrm{mg}$ doxycycline and $900 \mathrm{mg}$ rifampin and streptomycin $1 \mathrm{~g} /$ day for 21 days, all administered orally. One-week after the completion of the antibiotic treatment, his serologic tests and blood cultures were negative for brucella. To date, there has been no recurrence of his illness.

The rate of implanted cardiac device infections ranges from 0.8 to 5.7 per cent according to some reports (1). The infections may involve the pacemaker pocket or transvenous leads, or both. Pocket infections involve the subcutaneous pocket containing device and the sub-cutaneous segment of the leads. Deeper infections involving transvenous leads are associated with bacteraemia, endovascular infection and vegetation on the lead. Alternatively, device infections can be classified by the mode of infection. The device and/or pocket itself might be the primary source of the infection. Or the device may be seeded from bacteraemia secondarily. This is particularly true of gram-positive bacteraemia. But gram-negative organisms like brucella very rarely seed cardiac devices $(2,3)$. In the case presented here, the device was infected secondarily but later behaved as a primary source of infection causing the relapses of brucellosis. This case clearly demonstrates that if a patient with implanted cardiac device suffers from the relapses of brucella despite effective antibiotic treatment, the infection of the implanted device by brucella should be kept in mind.

Keywords: Brucellosis, Brucella melitensis, infection 
From: NS Yelgeç, AT Alper, M Keskin, AI Tekkeşin, C Türkkan

Correspondence: Dr NS Yelgec, Department of Cardiology, Siyami Ersek Cardiovascular and Thoracic Surgery Center, Istanbul 34668,Turkey.Email:yelgec@gmail. com

\section{REFERENCES}

1. Eggiman P, Waldvogel F. Pacemaker and defibrillator infection. In Waldvogel F, Bisno A (eds). Infections Associated with Indwelling Medical Devices. Washington: ASM Press, 2000: 247 64.

2. Uslan DZ, Sohail MR, Friedman PA. Frequency of permanent pacemaker or implantable cardioverter-defibrillator infection in patients with gram-negative bacteremia. Clin Infect Dis 2006; 43: 731-6.

3. Al-Majid FM. Pacemaker lead endocarditis due to Brucellosis. Saudi Med J 2010; 31: 448-50. 